\title{
The New and Evolving Roles of Sodium Glucose Co- Transporter 2 Inhibitors
}

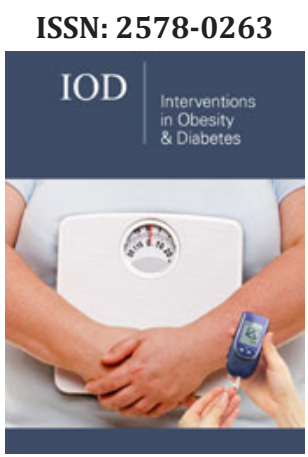

*Corresponding author: Brian K Irons, Department of Pharmacy Practice, Jerry $\mathrm{H}$ Hodge School of Pharmacy, Texas Tech University Health Sciences Center, Lubbock, USA

Submission: 監 June 05, 2020

Published: 悳June 22, 2020

Volume 4 - Issue 2

How to cite this article: Brian K Irons. The New and Evolving Roles of Sodium Glucose Co-Transporter 2 Inhibitors. Interventions Obes Diabetes 4(2). IOD.000585. 2020. DOI: $10.31031 /$ IOD.2020.04.000585

Copyright@ Brian K Irons. This article is distributed under the terms of the Creative Commons Attribution 4.0 International License, which permits unrestricted use and redistribution provided that the original author and source are credited.

\section{Brian K Irons*}

Department of Pharmacy Practice, Jerry H Hodge School of Pharmacy, Texas Tech University Health Sciences Center, USA

\begin{abstract}
Over the last five years there has been an increasing number of clinical trials demonstrating improved outcomes for sodium glucose co-transporter 2 inhibitors beyond improvements in glycemic control. The cardiovascular safety studies with empagliflozin, canagliflozin, and dapagliflozin showed varying degrees of improved cardiovascular outcomes in patients with type 2 diabetes and established cardiovascular disease or at high risk for such but each showed a reduction in heart failure admissions in this population. These studies also showed an improvement in renal outcomes. In 2019 canagliflozin demonstrated a reduction in the progression of diabetic kidney disease in patients with type 2 diabetes and existing nephropathy leading to the first new indication for the treatment of diabetic kidney disease in nearly two decades. Recently, dapagliflozin has shown to reduce cardiovascular death or heart failure admissions in patients with heart failure with reduced ejection fraction with or without the presence of diabetes.
\end{abstract}

Keywords: Cardiovascular disease; Type 2 diabetes; Sodium glucose co-transporter 2 inhibitors; Diabetic kidney disease; Heart failure

\section{Introduction}

In 2013 the Food and Drug Administration (FDA) approved the first sodium glucose cotransporter 2 inhibitor (SGLT2i), canagliflozin, for the treatment of hyperglycemia in patients with type 2 diabetes (T2D). This was followed a year later by the approvals of two other SGLT2is, empagliflozin and dapagliflozin. The last currently FDA approved agent in this class, ertugliflozin, was approved in late 2017. Following approval each agent had to also undergo further evaluation of their cardiovascular safety as mandated by the FDA at that time for all new agents used in the treatment of T2D hyperglycemia. The first cardiovascular outcome trial (CVOT) appeared in 2015 and showed that empagliflozin not only showed no increased cardiovascular risk but actually decreased cardiovascular and all-cause mortality in patients with both diabetes and established cardiovascular disease [1]. Subsequently in 2017 the CVOT of canagliflozin showed a $14 \%$ relative risk reduction in the combined outcome of nonfatal stroke, myocardial infarction (MI), or cardiovascular death in subjects with established cardiovascular disease or at high risk for such but did not show a reduction in cardiovascular mortality [2]. Last year, dapagliflozin's CVOT failed to demonstrate a cardiovascular benefit in the same combined outcome as the canagliflozin study [3]. No CVOT of these agents showed a significant reduction in MI or stroke but each showed a significant reduction in hospital admissions for heart failure. The CVOT for ertugliflozin was completed in late 2019 and results should be published in the near future. As a result of the CVOT for empagliflozin the FDA approved a new indication for the agent late in 2016 for the reduction of cardiovascular death in patients with T2D and cardiovascular disease. In the fall of 2018, the FDA approved dapagliflozin with a new indication to reduce MI, stroke, or cardiovascular death in patients with T2D and cardiovascular disease. The CVOTs have also led to updates in clinical practice guidelines recommending the use of agents within this class that have demonstrated reduced cardiovascular outcomes in patients with T2D and cardiovascular disease $[4,5]$.

\section{Mini Review}

Embedded within each currently published CVOT, secondary assessment of a composite renal outcome was also evaluated though the data interpretation is limited as the studies were not specifically designed to evaluate renal outcomes [6-8]. The composite renal outcome 
in each study differed somewhat but the end results showed a significant reduction in the outcome in each trial. More importantly, each trial showed a significant reduction in progression of renal function decline and not just an improvement in albuminuria as observed in the CVOTs involving glucagon-like peptide 1 agonists [9]. In 2019 the Canagliflozin and Renal Events in Diabetes with Established Nephropathy Clinical Evaluation (CREDENCE) was published and as the name suggests was designed to specifically evaluate robust renal outcomes in patients with T2D and existing nephropathy (baseline estimated glomerular filtration rate (gfr) $56 \mathrm{ml} / \mathrm{min} / 1.72 \mathrm{~m}^{2}$, and urine albuminuria $927 \mathrm{mg} / \mathrm{g}$ ) [10]. This study demonstrated a $30 \%$ relative reduction in the composite outcome of dialysis, kidney transplant, gfr $<15 \mathrm{ml} / \mathrm{min} / 1.72 \mathrm{~m}^{2}$, doubling of creatinine, or renal death and a $40 \%$ reduction in doubling of serum creatinine. The study also found a significant reduction in cardiovascular events and admissions for heart failure. As a result of this study, the FDA approved a new indication for canagliflozin in the fall of 2019 to treat diabetic kidney disease (DKD) and to reduce heart failure admissions in patients with DKD and diabetes. This is the first such approval by the FDA in nearly 20 years for the treatment of DKD. Earlier this year, the Dapagliflozin on Renal Outcomes and Cardiovascular Mortality in Patients with Chronic Kidney Disease (known as Dapa-CKD) was stopped earlier than anticipated after an interim analysis found a benefit of dapagliflozin over placebo in subjects with chronic kidney disease (gfr $25-75 \mathrm{ml} / \mathrm{min} / 1.72 \mathrm{~m}^{2}$ and albuminuria $200-5000 \mathrm{mg} / \mathrm{g}$ ) $[11,12]$. The primary composite outcome of this study was the first occurrence of at least a 50\% sustained decline in gfr, end stage renal disease, or renal death. Results are yet to be published but the most unique aspect of this study is that T2D was not an inclusion criteria.

2019 also showed a unique use of a SGLT2i in the Dapagliflozin and Prevention of Adverse Outcomes in Heart Failure (Dapa-HF) study [13]. The trial evaluated dapagliflozin compared to placebo in over 4700 patients with heart failure and reduced ejection fraction for an average of 18 months. The investigators found a $26 \%$ relative risk reduction in the primary composite outcome of worsening of heart failure or cardiovascular death. As in the Dapa-CKD study, T2D was not an inclusion criteria and improvement in the primary outcome was found regardless of diabetes status. Earlier this year the FDA approved a new indication for dapagliflozin to reduce the risk of cardiovascular death and hospitalization for heart failure in patients with heart failure with reduced ejection fraction. This is the first FDA approval for a SGLT2i for a non-diabetes disease state. Emplagliflozin is currently being investigated in patients with heart failure regardless of diabetes status in patients with heart failure with reduced and preserved ejection fraction.

\section{Conclusion}

The use of SGLT2is has evolved significantly over the last 7 years and the effects of this class of agent appears to go well beyond simply reducing elevated glucose concentrations in patients with T2D. There is data to support their use in reducing cardiovascular risk particularly in patients with established cardiovascular disease, reducing the progression of DKD, and improving outcomes in patients with or without diabetes in those with heart failure with reduced ejection fraction.

\section{References}

1. Zinman B, Wanner C, Lachin J, Fitchett D, Bluhmki E, et al. (2015) Empagliflozin, cardiovascular outcomes, and mortality in type 2 diabetes. N Engl J Med 373(22): 2117-2128.

2. Neal B, Perkovic V, Mahaffey K, de Zeeuw D, Fulcher G, et al. (2017) Canagliflozin and cardiovascular and renal outcomes in type 2 diabetes. N Engl J Med 377(7): 644-657.

3. Wiviott S, Raz I, Bonaca M, Mosenzon O, Kato E, et al. (2019) Dapagliflozin and cardiovascular outcomes in type 2 diabetes. N Engl J Med 380(4): 347-357.

4. American Diabetes Association (2020) Cardiovascular disease and risk management: Standards of medical care in diabetes -2020. Diabetes Care 43(Suppl 1): S111-S134.

5. Garber A, Handelsman Y, Grunberger G, Einhorn D, Abrahamson M, et al. (2020) Consensus statement by the American Association of Clinical Endocrinologists and American College of Endocrinology on the comprehensive type 2 diabetes management algorithm- 2020 executive summary. Endocrine Practice 26(1): 107-139.

6. Wanner C, Inzucchi S, Lachin J, Fitchett D, von Eynatten M, et al. (2016) Empagliflozin and progression of kidney disease in type 2 diabetes. $\mathrm{N}$ Engl J Med 375(4): 323-334.

7. Perkovic V, de Zeeuw D, Mahaffey K, Fulcher G, Erondu N, et al. (2018) Canagliflozin and renal outcomes in type 2 diabetes: results from the CANVAS Program randomised clinical trials. Lancet Diabetes Endocrinol 6(9): 691-704.

8. Mosenzon O, Wiviott S, Cahn A, Rozenberg A, Yanuv I, et al. (2019) Effects of dapagliflozin on development and progression of kidney disease in patients with type 2 diabetes: An analysis from the DECLARE-TIMI 58 randomised trial. Lancet Diabetes Endocrinol 7(8): 606-617.

9. Irons B, Minze M, Chastain L, McMurry M (2020) Updates in the treatment of diabetic kidney disease-a focus on incretin mimetics and sodium glucose co-transporter 2 inhibitors. J Endocr Disord 6(1): 1033.

10. Perkovic V, Jardine M, Bompoint S, Bompoint S, Heerspink H, et al. (2019) Canagliflozin and renal outcomes in type 2 diabetes and nephropathy. N Engl J Med 380(24): 2295-2306.

11. Astra Zeneca (2020) Farxiga Phase III DAPA-CKD trial will be stopped early after overwhelming efficacy in patients with chronic kidney disease.

12. Astra Zeneca (2020) A study to evaluate the effect of dapagliflozin on renal outcomes and cardiovascular mortality in patients with chronic kidney disease (Dapa-CKD). National Institute of Health, US National Library of Medicine, USA.

13. McMurray J, Solomon S, Inzucchi S, Kober L, Kosiborod M, et al. (2019) Dapagliflozin in patients with heart failure and reduced ejection fraction. N Engl J Med 381(21): 1995-2008. 